\title{
Zebrafish: A Marvel of High-Throughput Biology for 21st Century Toxicology
}

\author{
Sean M. Bugel • Robert L. Tanguay • Antonio Planchart
}

Published online: 7 September 2014

(C) Springer International Publishing AG 2014

\begin{abstract}
The evolutionary conservation of genomic, biochemical, and developmental features between zebrafish and humans is gradually coming into focus, with the end result that the zebrafish embryo model has emerged as a powerful tool for uncovering the effects of environmental exposures on a multitude of biological processes with direct relevance to human health. In this review, we highlight advances in automation, high-throughput screening, and analysis that leverage the power of the zebrafish embryo model for unparalleled advances in our understanding of how chemicals in our environment affect our health and wellbeing.
\end{abstract}

Keywords Zebrafish embryo model · High-throughput screening $\cdot$ Environmental exposure $\cdot$ Human health

\section{Introduction}

The last 20 years witnessed the rate of studies using the zebrafish to elucidate mechanisms of vertebrate gene function increase exponentially, from approximately five publications in 1990 to over 2,500 in 2013. During this interval, a more modest increase in the publication of zebrafish-based toxicology studies was underway. However, with the new millennium came a new trend and we are in the midst of a shift away from using zebrafish exclusively for developmental studies and towards leveraging their many advantages to understand processes by which environmental exposures influence

S. M. Bugel $\cdot$ R. L. Tanguay

Department of Environmental and Molecular Toxicology, Oregon

State University, Corvallis, OR, USA

A. Planchart $(\square)$

Department of Biological Sciences and Center for Human Health and

the Environment, North Carolina State University, Campus Box

7617, Raleigh, NC 27695, USA

e-mail: antonio_planchart@ncsu.edu development and disease (Fig. 1). The reasons for this shift are complex and somewhat predicated on the need to move from in vitro to in vivo studies while adhering to principles that reduce, replace, or refine the use of animals in research [1]. As a result of this revolution, leveraging zebrafish to investigate the most pressing biological problems of human development, disease, and the role environmental exposures play in adverse biological outcomes is made possible, often at modest costs. In the past couple of years, reviews describing the potential use of zebrafish in toxicology research have been published [2-7]; therefore, this review, while not seeking to be comprehensive, aims to summarize the last few years of toxicology research in which zebrafish made significant contributions. We focus on results from a number of screening experiments, which are gradually informing our understanding of the complex interplay between environmental perturbation and human health and wellbeing.

\section{Zebrafish and Human Embryos: Living in a Fishbowl}

Zebrafish experience a dramatically different environment than humans except during the embryonic period, during which human embryos develop in an aquatic environmentthe amniotic fluid. The origins of amniotic fluid can be subdivided into pre-placentation and post-placentation [8]. Pre-placentation amniotic fluid derives from maternal plasma and enters the extracoelomic cavity by passive and active mechanisms, whereas post-placentation amniotic fluid originates in the embryo, from which it is exuded through prekeratinized skin, urinated or (to a lesser extent) defecated. However, all amniotic fluid has its origins in maternal plasma, which transports nutrients, electrolytes, and water to the embryo, as well as any toxicants and xenobiotics that may be present in maternal circulation and are able to diffuse across the placenta. Not surprisingly, 'waterborne' exposures to 


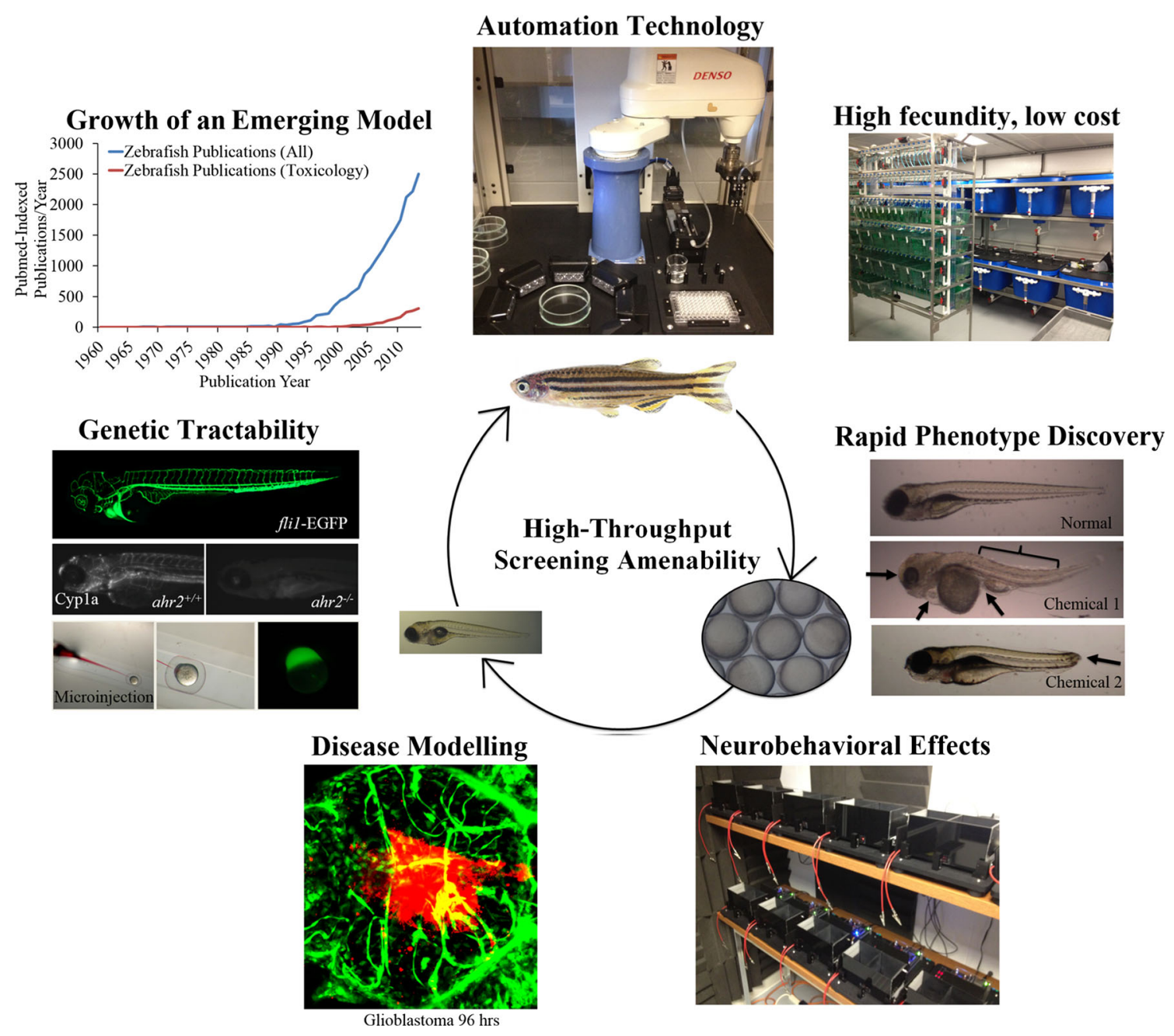

Fig. 1 Zebrafish are an emerging vertebrate model for HT toxicity screening, disease modeling, phenotype discovery, and chemical mechanisms of action. Their high fecundity, low cost, and rapid development make nearly all in vivo biological assays amenable to HT studies. As technologies, the zebrafish has rapidly become one of the premier vertebrate models for biological discovery. HT high-throughput

environmental factors occur; the presence of xenobiotics [9, 10], industrial pollutants [11, 12], medications [13], chemicals in household items $[14,15]$, and chemicals derived from lifestyle habits $[16,17]$ have been isolated from human amniotic fluid, which is swallowed, breathed in, and recycled by the developing fetus beginning at about week 10 and continuing throughout gestation [8]. Consequently, the absorption routes during embryogenesis in humans are probably similar to those in zebrafish, including dermal, gastrointestinal, and respiratory, although this has not been rigorously tested.

Performing waterborne exposures in the zebrafish embryo model is advantageous for several reasons:

1. Large numbers of zebrafish embryos can be exposed simultaneously in relatively small volumes (e.g. $>10 \mathrm{em}-$ bryos per $\mathrm{ml}$ ), generating a robust sample for downstream applications, including transcriptomics, proteomics, and metabolomics.

2. The zebrafish chorion, an acellular envelope surrounding the embryo and riddled with pores between 0.5 and $0.7 \mu \mathrm{m}$ in diameter [18], is highly permeable to a wide range of small molecules and xenobiotics. Instances in which the chorion is an effective barrier can be overcome by automated enzymatic dechorionating processes.

3. Short-duration exposures from $1 \mathrm{~h}$ to a few days will intersect with multiple developmental processes due to the accelerated growth rate of zebrafish embryos relative to humans (from fertilized egg to free-swimming hatchling in 3-5 days), possibly mimicking chronic exposures of weeks to months in humans.

4. Exposures timed to coincide with very specific developmental endpoints/events are possible.

For these reasons, and others discussed in the following sections, the zebrafish is imbued with the ability to enhance our understanding of potential risks of exposure to diverse 
environmental challenges under a variety of experimental conditions.

\section{Zebrafish: A Molecular 'Swiss Army' Knife}

There are many advantages to using zebrafish for reductionist and systems biology, and for low-throughput (LT) and highthroughput (HT) toxicology research. Genetically, zebrafish are very similar to humans - approximately $70 \%$ of human protein-coding genes have orthologs in the zebrafish, and over $80 \%$ of human disease-associated genes have a zebrafish counterpart [19]. Furthermore, an ancestral genome duplication in teleosts led to the formation of ohnologs (paralogs that arise from whole genome duplication [20]) that in many cases underwent subfunctionalization [21], i.e. the ancestral function 'split' among ohnologs; therefore, the resolution of effects of environmental exposures on the mechanics of gene and/or protein function is often higher in zebrafish. An excellent example of this is found in the $S O X 9$ locus, encoded by one gene in humans but two-sox $9 a$ and $\operatorname{sox} 9 b$-in the zebrafish [22]. In addition to its role in vertebrate male sex determination [23], SOX9 is required for chondrogenesis [24]. Proliferation, differentiation, and condensation of chondrocytes are regulated by SOX9 in humans, whereas in zebrafish, proliferation and differentiation are regulated by sox $9 b$ and condensation by $\operatorname{sox} 9 a$ [22]. It was subsequently discovered that exposure to 2,3,7,8-tetrachlorodibenzo- $p$-dioxin (TCDD) impairs vertebrate chondrogenesis by interfering with the expression of $\operatorname{sox} 9 b$ but not $\operatorname{sox} 9 a$ [25]; thus, proliferation, but not condensation, is affected, which is consistent with the TCDD-induced craniofacial phenotype. Other examples of differential response by ohnologs to environmental factors have been reported [26].

Zebrafish have the capacity to spawn hundreds of developmentally synchronized embryos in a single spawning event. Embryos are small (diameter $\leq 1 \mathrm{~mm}$ ), optically transparent, develop in an open environment, and are easily manipulated-properties that can be exploited in robotics-driven arraying schemes without resorting to complicated culturing protocols and sterile environments. Chemical screen assays can be implemented using in vitro-equivalent protocols, and screening large numbers of embryos simultaneously ensures sufficient replicates and robust statistical power. Existing and future integrated technologies will permit measuring multiple parameters during the course of an experiment, including growth rate, morphogenesis, behavior, morbidity and mortality, and gene and protein expression profiles. Multiple transgenic lines expressing fluorescent markers under cell- and tissue-specific promoters are being leveraged to study specific molecular pathways, often in a single-cell lineage or tissue, and to generate rapid mechanistic insights into the role of chemical exposures on biological function [27].
The transparency of zebrafish embryos opens opportunities previously unattainable in vertebrates. For example, lightsheet microscopy coupled with genetically engineered fluorescent zebrafish permit high-resolution imaging of cell movements during embryonic development while environmental conditions are altered in a controlled manner [28]. Therefore, the molecular and cellular basis of environmentally-induced developmental defects rooted in cell migration, for example, can be assayed in the context of a whole embryo in real time.

Forward [29, 30] and reverse [31, 32, 33••] genetic screens are now routine laboratory techniques in the zebrafish, and multiple consortia have ongoing projects to identify novel nonsense mutations across the entire zebrafish exome by TILLING (Targeting Induced Local Lesions in Genomes) [34], which are made available to investigators as heterozygous carriers for a nominal fee. The greatest advantage of using the zebrafish for these applications is cost; at a fraction of the cost of making a similar mutation in a mouse, the zebrafish is the most economical model organism in which to examine vertebrate gene function.

\section{Zebrafish: Spawning a Revolution in In Vivo High-Throughput (HT) Screening}

Over 80,000 chemicals are manufactured and used worldwide [35] in countless consumer products, including natural and processed foods; infant products, toys and clothing; furniture and housewares; building materials, and the workplace. In the US, enactment of the Toxic Substances Control Act of 1976 began an effort to regulate chemicals but exempted thousands of existing chemicals, and, consequently, it has generally been regarded as weak [36, 37]. Unlike the EU's REACH (Registration, Evaluation, Authorization and Restriction of Chemicals) regulation [38], which mandates that manufacturer's provide adequate scientific evidence of a chemical's safety in human affairs if its manufacture or importation exceeds 1 metric ton, no formal regulatory mechanism exists in the US to ensure that chemicals conform to rigorous biological safety standards. Thus, scientific and lay communities are largely ignorant of the effect chemical exposures have on our health and well-being, with a few notable exceptions, such as polychlorinated biphenyls [39] and thalidomide [40]. Considering that most often we come into contact simultaneously with groups of chemicals, the number of possible combinations is astronomical (e.g. over 3 billion pairwise combinations of chemicals in an arsenal of 80,000), illustrating the daunting road ahead if we are to embark on understanding how environmental exposures of anthropogenic origin affect human development and health and the health of our planet.

Understanding how individual chemicals and their metabolites alter complex biological processes requires large-scale, 
HT analytical studies, which are often performed in vitro using well-characterized cell lines of different origins-derived from different organs, ethnicities, sexes, and populations. In addition, HT chemical screens require simplicity, speed, assay validity, robustness, and statistical power in order to succeed, and these have been the driving forces behind in vitro cellbased systems. However, it is often very difficult to establish concordance between a chemical's effect in an in vitro cellbased two-dimensional assay and its role in human disease because of the complexity inherent in an animal: dimensionality; multiple organ systems; broad spectrum of cell types and physiology, including sessile versus motile cells, transport mechanisms, metabolic routes, and complexity of extracellular matrices; and differences in gene expression, including epigenetic variation and allelic composition. Advances in three-dimensional cell-based systems [41, 42] and threedimensional prototype organs [43] have the potential to overcome some of these barriers to translation from in vitro results to in vivo predictions but we would argue that most are not amenable to modeling with current technology, neither in real nor virtual realms, because they lack the complexity, interorgan interactions, and structural organization characteristic of whole animals.

Under current paradigms, in vitro HT assays are the first line of inquiry to identify high-priority chemicals from the $>80,000$ in production because of the speed with which the assays can be performed. These assays are then followed by in vivo studies on a significantly reduced chemical space using model organisms, principally rodents, to identify the most acute and chronically pernicious chemicals that merit further investigation and possibly regulatory oversight. Unfortunately, this latter approach is very inefficient since the vast majority of chemicals cannot be tested in a timely manner and experiments with rodents are expensive. However, for reasons we enumerated earlier, a transition to the zebrafish as the first line of HT chemical screening could resolve many of these issues. This approach has gained traction at the US Environmental Protection Agency through its ToxCast program, in which the zebrafish embryo model played a significant role during the phase I analysis [44]; a subsequent study in the zebrafish with all 1,060 unique ToxCast phase I and II compounds has also been reported by one of the authors (RLT [45•]), in which 487 (46\%) compounds were seen to elicit significant adverse outcomes on development at environmentally relevant concentrations.

In the following sections, we describe some of the recent results of HT and scalable LT chemical screens using the zebrafish embryo as an alternative to in vitro cell-based HT assays with the aim of demonstrating that the zebrafish embryo rivals the best in vitro systems and contextualizes, in a complex organism, the effects of chemical and environmental exposures on development and disease.

\section{Developmental Toxicity}

Taking advantage of the zebrafish's external and transparent development, the zebrafish embryo model has been exploited in a wide array of developmental toxicology studies, including skeletal development [46, 47], immune development [48], neurodevelopment $[49,50]$, cardiovascular development [51-53], and regeneration [54-57]. A recent spate of screens have used the zebrafish embryo model to analyze potential developmental toxicity of small molecule libraries previously uncharacterized in in vivo models [44]: unknown contaminants in landfill soil [58]; nanomaterials (reviewed by Fako and Ferguson [59]); and ototoxic drugs [60]. Recent studies illustrate that the zebrafish embryo model can be used to screen and prioritize compounds suspected as human developmental toxicants [61]. In the recent past, limitations of chemical screening with zebrafish were largely due to laborious experimental setup and animal/chemical handling; data acquisition; and processing. However, in recent years, major strides have been made in automation technology and highcontent image analysis. Therefore, developing pipelines and standardized methods for developmental toxicity screening is now possible for large chemical libraries [62]. While many specialized endpoints for toxicity exist (reviewed in other sections), an array of standardized phenotypes for general developmental toxicity assessments in zebrafish, including teratogenic effects, has been developed. These standardized phenotype screens enable measures of teratogenicity and malformation observed in a variety of embryonic structures, including notochord, yolk-sac, heart, body axis, eyes, snout, jaw, otic vesicle, brain, somites, trunk, pectoral and caudal fins, pigmentation, circulatory system, and swim bladder. Other measurable endpoints include mortality, spontaneous motion, developmental stage progression, and touch response. Observable phenotypes occurring as a result of chemically perturbed development are highly diverse and may be pleiotropic or polygenic depending on a chemical's mode of toxicity. Characterizing a chemical's developmental toxicity phenotype(s) is a necessary first step in the design of mechanistic studies, which can utilize reverse and forward genetics to determine a specific receptor or molecule's role in the phenotype. For example, developmental toxicity of TCDD has been studied extensively; TCDD toxicity generally includes pericardial and yolk-sac edemas [63, 64]. Mechanistic studies anchored to this phenotype in zebrafish have allowed for the dissection of the AHR pathway and its function in developmental toxicity $[65,66]$. Due to advances in automation, highcontent imaging, and forward/reverse genetics, phenotypebased chemical and mechanistic screens have placed zebrafish at the forefront of in vivo chemical screening. The utility of zebrafish in HT developmental toxicity screening will undoubtedly be invaluable for predictive toxicology for many years to come. 


\section{Cardiotoxicity}

Studies of cardiotoxic effects from chemical exposures on the developing vertebrate heart are challenging because most vertebrates die in the absence of normal heart development. Zebrafish, on the other hand, survive up to 5 days postfertilization (dpf) in the complete absence of a functional cardiovascular system, even though a functional heart normally develops within the first $24 \mathrm{~h}$ of life; therefore, exposuredriven cardiotoxic effects can be studied across the spectrum of heart development, including initial stages of cardiac mesoderm specification, patterning of the heart, and establishment of cardiac electrical conduction (see Staudt and Stainier for a review of zebrafish heart development [67]). Although the zebrafish heart is a two-chambered organ, many developmental and molecular landmarks are evolutionarily conserved in zebrafish and humans, including sarcomere formation [68], atrioventricular septum development [69], and apex-to-base ventricular activation patterns [70]. In addition, numerous mutations associated with human congenital heart defects and cardiomyopathies have been uncovered as a direct result of cardiovascular research performed in zebrafish (for review, see Bakkers [71]).

The zebrafish embryo model has anchored numerous cardiotoxicity studies. For example, studies of complex mixtures such as those found in oil spills (Alaska North Slope crude oil from the Exxon Valdez, and Mississippi Canyon 252 oil from the Deep Water Horizon) [72] identified polycyclic aromatic cardiotoxicants in zebrafish that induced cardiac edema, defective heart looping, hemorrhage, and reduction in arteriovenous circulation, which were consistent with observations by others that activation of the aryl hydrocarbon pathway by dioxins and dioxin-like substances leads to gross morphological and functional abnormalities in the zebrafish heart [73-76]. In other studies, retinoic acid and TCDD, both well-characterized cardiotoxicants, activated a common transcriptional response via distinct, non-overlapping sets of genes associated with heart failure [77].

The zebrafish embryo model has been successfully deployed to screen for cardiotoxic effects elicited by carbaryl and valproic acid [78], atypical antipsychotic drugs [79], kinase inhibitors [80], human cardiotoxic drugs [81], and chemotherapeutics [82], thus illustrating the value of zebrafish as a preclinical testing paradigm to predict cardiotoxic effects elicited by drugs under development. Recently, in vivo analysis of proliferating cardiomyocytes (the functional unit of cardiac muscle) in zebrafish embryos was leveraged to identify chemicals that could promote cardiomyocyte proliferation, thus opening a window into the possible therapeutic replacement of infarct-damaged cardiac tissue in humans [83].

Transgenic lines in which specific cardiac cell types are fluorescently labeled, including $c m l c 2: E G F P$ (differentiated cardiomyocytes [84]), gata4:EGFP (regenerative cardiomyocytes [85]), pard3:EGFP (endocardium [86]), and tcf21:DsRed (epicardium [87]), are available. These and other fluorescent lines are valuable resources with which to study the effects of chemical exposure on heart development with unprecedented granularity, including understanding the effects of cardiotoxicants in a cell-specific context. Coupled with HT automated visualization and sorting analysis, there is no better model for cardiotoxic research than the zebrafish embryo.

\section{Hepatotoxicity}

Liver toxicity is often a top concern during drug and pharmaceutical development and for chemical risk assessment. To evaluate hepatotoxicity, rodent liver assays are typically used but generally tend to be LT because studies are relatively expensive and slow, and as a result they suffer from low sampling sizes. In contrast, developing zebrafish can be utilized for hepatotoxicity screening, which is amenable to HT applications because of their transparency, speed of development, large sample sizes, and low cost. Hepatotoxicity studies in the zebrafish can therefore be conducted earlier in drug development, which may aid the decision-making process during research and development. There are a number of early indicators of liver toxicity in zebrafish [88].

Embryonic zebrafish develop a rudimentary liver by $24 \mathrm{~h}$ post-fertilization (hpf), which undergoes rapid growth and differentiation to become fully functional by $72 \mathrm{hpf}$ (reviewed by $\mathrm{Chu}$ and Sadler [89]). Specialized endpoints can be screened in an HT manner by taking advantage of automated technologies, high-content imaging, transgenic lines, or assays for liver-specific enzymes. Similar to mammals, zebrafish respond to xenobiotics and oxidative stress with induction of phase I and II drug metabolism genes. Furthermore, there is a high degree of similarity between zebrafish and human cytochrome P450 (CYP) gene sequences and protein function [90] and the majority are expressed throughout development, which allows developing zebrafish to be used for toxicological and pharmacological studies. Expression of genes involved in drug metabolism can be visualized in the transparent embryonic and larval zebrafish, using transgenic strains or whole-mount in situ hybridization (WISH). For example, transgenic zebrafish expressing green fluorescent protein (GFP) regulated by the CYP1A promoter can be used as a rapid, non-destructive in vivo screen for induction of phase I metabolism [91, 92]. Other rapid in vivo non-destructive techniques that are amenable to HT screening include the ethoxyresorufin-O-deethylase (EROD) assay, which can be visualized and quantified by fluorescence microscopy [93]. Using this technique, many chemicals can be screened for CYP-specific activity. Additional assays, including high-resolution microscopy, liver morphometrics, apoptosis and necrosis, and other histopathological endpoints, 
are readily applied using non-destructive methods. Advances in automation and software analysis have made screening these endpoints achievable in HT applications.

\section{Endocrine Disruption and Reproductive Toxicity}

No large-scale studies of endocrine disruption and reproductive toxicity have yet to utilize zebrafish, although endocrine disruption assays and reproductive toxicity screens in the zebrafish are amenable to HT applications. All major hormone receptors in humans have functionally conserved zebrafish orthologs that are active in developing zebrafish (e.g. thyroid [94], androgen [95], estrogen [96], and gonadotropin [97] receptors). Although understanding of sexual differentiation in zebrafish remains nebulous [98], gametogenesis is similar between humans and zebrafish $[99,100]$. We anticipate that in the near future new and current standardized methods will come online for HT endocrine and reproductive toxicity screening in zebrafish; we predict that once these assays are available, zebrafish will be invaluable for identifying reproductive toxicants and novel endocrine disruptors. Using the zebrafish model as an in vivo whole animal endocrine screening tool offers advantages over in vitro cell-based screens and in vivo rodent studies, which suffer from low complexity and LT/high cost, respectively.

Endocrine-disrupting chemicals can be screened in vivo in a cost-efficient manner using rapid assays; when coupled to gene analysis studies, pathway-specific effects of exposure are readily measurable. There are many endocrine biomarkers useful for screening pathway-specific effects in developing zebrafish. For example, VTG (vitellogenin) and CYP19a1b (brain aromatase) genes are sensitive estrogen receptorregulated biomarkers that are inducible in embryonic-larval zebrafish by xenoestrogens [101]. These biomarkers, and others, can be evaluated qualitatively by in situ hybridization, or quantitatively by real-time polymerase chain reaction (PCR). For example, in a rapid 4-day zebrafish embryolarval bioassay, VTG messenger RNA (Mrna) expression was highly induced by estrogen, and completely inhibited (>95\% inhibition) by co-exposure to several dioxins, including TCDD [102], thereby demonstrating that the embryolarval bioassay can be used to screen for potential endocrine system agonists and/or antagonists. The use of gene-specific mRNAs as biomarkers is largely a matter of arraying embryos, performing exposures, lysing embryos, extracting RNA, and coupling complementary DNA (cDNA) synthesis with PCR amplification; these steps are readily amenable to automation, which could speed the discovery of novel biomarkers when coupled with libraries of gene-specific primers. Other methodologies exploit the ability to make transgenic zebrafish that express GFP controlled by any promoter one chooses. One example - a transgenic in which the CYP19alb promoter drives expression of GFP — was recently developed for rapid screening of estrogen mimics by measuring changes in fluorescence following exposure [103]. Currently, HT cell-based in vitro assays are commonly used to explore pathwayspecific endocrine activities for large chemical libraries by deploying assays such as the CALUX assay [104]. We predict that, in the near future, the zebrafish embryo model will become the benchmark test for uncovering endocrinedisrupting compounds in in vivo screens at costs that rival traditional cell-based assays.

Although there are minor differences, zebrafish are an excellent substitute for mammalian reproductive toxicity studies because their reproductive processes are comparable to those observed in mammals. Developmentally, zebrafish and rodents require approximately the same time from conception to reproductive maturity ( $\sim 3$ months). Zebrafish primordial germ cells are migratory and detectable prior to $24 \mathrm{hpf}$. Their gonads, like all vertebrate gonads, are initially bipotential and develop into ovaries or testes between 21 and $45 \mathrm{dpf}$. This critical window of sexual differentiation is sensitive to reproductive toxicants that may disrupt gonadogenesis and result in reproductive deficits later in life. For example, exposure to TCDD during sexual differentiation in zebrafish results in reproductive toxicity later in life in males and females, which is also transgenerationally heritable $[105,106,107 \bullet]$. A large number of endpoints to evaluate effects on reproduction, whether exposures take place during sexual differentiation or in the adult, can be used, including fecundity, gonad histopathology (follicular development and progression, atresia, apoptosis, presence of ovotestis), organ morphometrics, reproductive biomarker expression (e.g. vitellogenin), embryo viability, and developmental toxicity in offspring of exposed adults.

The goal of screening chemicals for endocrine activity is to uncover those that may pose a risk to development and reproduction; moving towards an HT whole animal model is the best way to comprehensively evaluate these endpoints. HT screening in zebrafish far exceeds what can be done in rodent models at a fraction of the cost.

\section{Neurotoxicity}

The zebrafish is an excellent model for neurobiology studies, especially during the initial phases of nervous system development. Many examples of transgenic zebrafish expressing fluorescent reporters under the control of neural-specific promoters have allowed researchers unparalleled access to realtime investigations of neural architecture and function, thus paving the way towards comprehensive assessments of the effects of environmental exposures on diverse neurological endpoints, including molecular, cellular, anatomical, and functional phenotypes. Over the last 2 years, neural-specific 
transgenic zebrafish have been at the forefront of chemical screens for compounds targeting the circadian clock [108], dopaminergic pathway [109], neurogenesis [110], spontaneous activity [111], pleotropic neurotoxicity [112], and environmental influences on axon growth and connectivity [113].

Advances in adapting zebrafish for use in Parkinson's research [114], amyotrophic lateral sclerosis and frontotemporal lobar degeneration [115-119], mitophagy and neurodegeneration (reviewed by Wager and Russell [120]), and understanding how drugs affect locomotor function [121] open promising avenues of research exploring the link between occupational-environmental exposures and neurological disorders, in which some degree of risk may be attributed to early life exposures. To this end, behavioral screens are underway in several laboratories (including the authors'), in which chemical exposures coupled with transgenics and neurophysiological measurements aim to elucidate the molecular and cellular basis of chemically-induced behavioral abnormalities at sublethal doses. These studies are also designed to address the long-term consequences of early-life exposures on nervous system function.

\section{Nanotoxicity}

Nanotechnology is a rapidly evolving field, and nanomaterials are increasingly used commercially for a number of products and applications. A nanomaterial is an object in which one of the three dimensions is between 1 and 100 nanometers (nm). Nanomaterials often exhibit complex and unusual physical, chemical, and biological properties compared with largerscale materials, including differences in conduction, chemical reactivity, strength, and affinity to biological structures. Development of nanoparticle libraries (objects that scale between 1 and $100 \mathrm{~nm}$ on all three axes) have been reported [122] but have not been fully characterized for toxicity or evaluated for risk assessment in vivo. While no standard methods for evaluating nanotoxicity have been established, the zebrafish larval bioassay has been used to rapidly evaluate how physical properties such as size, shape, charge, and surface chemistry affect toxicity [123]. However, nanoparticle-specific exposure studies in zebrafish, including cadmium [124, 125], gold [126, 127], silver [127-132], silica [133], and titanium dioxide [134, 135], have been conducted to characterize physicochemical properties and correlate these with toxicity. The Nanomaterial-Biological Interactions Knowledgebase (NBI; http://nbi.oregonstate.edu/) is a repository of annotated and integrated data of nanomaterial characterization for which zebrafish have made substantial contributions. The sheer diversity of nanomaterials, combined with the pace at which they are being manufactured and utilized worldwide, requires using a model such as the zebrafish embryo for uncovering nanomaterial-biological interactions and potential mechanisms of toxicity.

\section{Role of Automation in HT Screening}

Advances in genetics and developmental biology have made the zebrafish the ideal small animal model to use for HT phenotype screening. However, one major bottleneck for chemical screening with zebrafish is embryo sorting and handling, and phenotype scoring. To overcome these major issues, advancement in two technologies will support the application of zebrafish in HT screens. The first is automation of embryo handling and sorting. The second is automation of phenotype-based endpoints using high-powered image analysis.

There are several handling steps required for chemical screening in zebrafish embryos that immediately need to be addressed for large-scale studies. Production of large clutches of embryos is relatively simple using mass embryo production systems, which can generate tens of thousands of embryos per day. However, manually sorting, dechorionating, and arraying clutches of this size for HT chemical screening is burdensome and time-consuming. Automation of these steps can free-up personnel time for other pressing tasks, which leads to increased throughput and productivity and improved consistency of animal handling [136]. Rapid dechorionation of large embryo clutches using enzymatic digestion and automated shaking/rinsing is possible. Using advanced image-based robotics, dechorionated embryos can be arrayed into single wells of a 96-well plate, allowing thousands of animals to be plated per day. These advancements in robotic automation have increased the throughput of chemical screening severalfold. Using these techniques, a single laboratory can conduct concentration-response studies for developmental toxicity with dozens of chemicals per day. This level of in vivo throughput is unrivaled by any other vertebrate model. The robotics for dechorionating and arraying are not currently commercially available, although several laboratories have implemented these technologies using commercially available robotic arms and machine-vision cameras to build custom instruments in-house. The automation of dechorionating and arraying lends itself to other downstream robotic applications, such as liquid handlers for automation of chemical exposures. Another emergent area of automation expected in the near future is large-scale microinjection of single-cell embryos. Currently, manual sorting and microinjection makes this technique impractical for HT applications. However, using automated microinjection, researchers can control dosing of chemicals, mRNA, or morpholinos to test specific hypotheses in an HT manner. The technology using modern robots to automate handling, arraying, and microinjecting is available but has not yet deployed in an HT context. 
With advancements in automation of animal handling, a concomitant advance in phenotype screening is also necessary to ensure HT capability. Automated image-based screens are emerging for phenotype discovery. To accomplish this, highresolution imaging to acquire photomicrographs of entire 96well plates is being developed. Software-based algorithms can scan high-content images and detect phenotypes (e.g. pericardial edema, yolk-sac edema, axial defects, etc.) [137, 138, 139॰]. However, to date, most large-scale chemical screens have relied on manual phenotype observation and analysis. While manual screening does have advantages, such as discovery of novel phenotypes, it is impractical for HT screening. Powerful customizable and trainable software algorithms will be required to automatically assess morphology parameters, including processing and analyzing large datasets. Overall, automation of handling and phenotype-based screening has vastly improved in recent years. Incremental improvements will increase the complexity of phenotypes that can be detected during automated screening and ultimately increase HT screening capabilities.

\section{Pharma Adoption of the Zebrafish Embryo Model: Swimming in Savings}

In 2010, the cost of bringing a drug to market in the US was estimated at $\$ 1.8$ billion [140], requiring approximately 9 years of research and development before transitioning to the bedside. Drug discovery requires multiple rounds of in vitro assays, animal testing, and clinical trials before receiving regulatory approval if deemed safe and efficacious. A significant cost of bringing drugs to market is associated with animal testing, which includes safety assessments, toxicity testing, and pharmacokinetic studies to determine absorption rates, distribution, metabolism and excretion (ADME), which are performed in expensive mammalian animal models. To reduce the use of mammals in drug research for ethical and economic reasons, especially during the initial phase of safety assessment and in vivo toxicity testing, the use of the zebrafish embryo model has garnered early success (reviewed by Zon and Peterson [141]).Significantly, screening zebrafish embryos with panels of biologically active compounds uncovered 16,16-dimethyl prostaglandin $E_{2}$ as an important modulator of vertebrate hematopoietic stem cell homeostasis [142]. As a result, a novel compound is now in phase II clinical trials for the treatment of hematologic malignancies in humans (http://clinicaltrials. gov). It stands to reason that economies of scale can be realized using the zebrafish in drug discovery. Consequently, a consortium of multinational pharmaceutical companies initiated the process of validating the zebrafish for use in their drug discovery pipeline [143].

\section{Conclusions}

Deciphering the effects of chemical exposures on human health and the environment is a daunting task that lies ahead for those interested. It will require new technologies, new assays, and more efficient ways of analyzing and imaging complex and large data sets. The zebrafish is, by all measures, a powerful model that is facilitating rapid advances in these three areas, all of which will require prolonged efforts akin to swimming upstream for a long time. This is not a bad thing; after all, "A dead fish can float downstream but it takes a live one to swim upstream" (W.C. Fields).

Acknowledgments This article was supported by NIH R01 ES016896 (RLT) and North Carolina State University (AP). To authors whose work was omitted, we can only offer our sincere apologies.

\section{Compliance with Ethics Guidelines}

Conflict of Interest Dr. Sean M. Bugel and Dr. Robert L. Tanguay each declare no potential conflict of interest. Dr. Antonio Planchart is in a domestic partner relationship with the Section Editor, Dr. Carolyn Mattingly.

Human and Animal Rights and Informed Consent This article does not contain any studies with human or animal subjects performed by any of the authors.

\section{References}

Papers of particular interest, published recently, have been highlighted as:

- Of importance

•. Of major importance

1. Anonymous. Reduce, refine, replace. Nat Immunol. 2010;11(11): 971.

2. Bailey J, Oliveri A, Levin ED. Zebrafish model systems for developmental neurobehavioral toxicology. Birth Defects Res C Embryo Today. 2013;99(1):14-23.

3. Gibert Y, Trengove MC, Ward AC. Zebrafish as a genetic model in pre-clinical drug testing and screening. Curr Med Chem. 2013;20(19):2458-66.

4. Lin S, Zhao Y, Nel AE, Lin S. Zebrafish: an in vivo model for nano EHS studies. Small. 2013;9(9-10):1608-18.

5. Helenius IT, Yeh JR. Small zebrafish in a big chemical pond. J Cell Biochem. 2012;113(7):2208-16.

6. Peterson RT, Macrae CA. Systematic approaches to toxicology in the zebrafish. Annu Rev Pharmacol Toxicol. 2012;52:433-53.

7. Sipes NS, Padilla S, Knudsen TB. Zebrafish: as an integrative model for twenty-first century toxicity testing. Birth Defects Res C Embryo Today. 2011;93(3):256-67.

8. Underwood MA, Gilbert WM, Sherman MP. Amniotic fluid: not just fetal urine anymore. J Perinatol. 2005;25(5):341-8.

9. Engel SM, Levy B, Liu Z, Kaplan D, Wolff MS. Xenobiotic phenols in early pregnancy amniotic fluid. Reprod Toxicol. 2006;21(1):110-2. 
10. Foster WG, Chan S, Platt L, Hughes Jr CL. Detection of phytoestrogens in samples of second trimester human amniotic fluid. Toxicol Lett. 2002;129(3):199-205.

11. Miller MF, Chernyak SM, Domino SE, Batterman SA, LochCaruso R. Concentrations and speciation of polybrominated diphenyl ethers in human amniotic fluid. Sci Total Environ. 2012;417-418:294-8.

12. Luzardo OP, Mahtani V, Troyano JM, et al. Determinants of organochlorine levels detectable in the amniotic fluid of women from Tenerife Island (Canary Islands, Spain). Environ Res. 2009;109(5):607-13.

13. Nolte DL, Mason JR. Maternal ingestion of orthoaminoacetophenone during gestation affects intake by offspring. Physiol Behav. 1995;58(5):925-8.

14. Chen M, Edlow AG, Lin T, et al. Determination of bisphenol-A levels in human amniotic fluid samples by liquid chromatography coupled with mass spectrometry. J Sep Sci. 2011;34(14):1648-55.

15. Ikezuki Y, Tsutsumi O, Takai Y, Kamei Y, Taketani Y. Determination of bisphenol A concentrations in human biological fluids reveals significant early prenatal exposure. Hum Reprod. 2002;17(11):2839-41.

16. Brien JF, Loomis CW, Tranmer J, McGrath M. Disposition of ethanol in human maternal venous blood and amniotic fluid. Am J Obstet Gynecol. 1983;146(2):181-6.

17. Divers Jr WA, Wilkes MM, Babaknia A, Yen SS. Maternal smoking and elevation of catecholamines and metabolites in the amniotic fluid. Am J Obstet Gynecol. 1981;141(6):625-8.

18. Schmidt W, Pfaller K, Klima G. Optical and electron microscopy studies of human fetal membranes. 2. Chorion and decidua [in German. Zentralbl Gynakol. 1982;104(18):1137-48.

19. Howe $\mathrm{K}$ et al. The zebrafish reference genome sequence and its relationship to the human genome. Nature. 2013;496(7446):498 503.

20. Ohno S. Evolution by gene duplication. Berlin: Springer-Verlag; 1970. p. xv. 160 p.

21. Taylor JS, Raes J. Duplication and divergence: the evolution of new genes and old ideas. Annu Rev Genet. 2004;38:615-43.

22. Yan YL, Willoughby J, Liu D, et al. A pair of Sox: distinct and overlapping functions of zebrafish sox9 co-orthologs in craniofacial and pectoral fin development. Development. 2005;132(5):1069-83.

23. Kent J, Wheatley SC, Andrews JE, Sinclair AH, Koopman P. A male-specific role for SOX9 in vertebrate sex determination. Development. 1996;122(9):2813-22.

24. Bi W, Deng JM, Zhang Z, Behringer RR, de Crombrugghe B. Sox9 is required for cartilage formation. Nat Genet. 1999;22(1): 85-9.

25. Xiong KM, Peterson RE, Heideman W. Aryl hydrocarbon receptor-mediated down-regulation of sox $9 \mathrm{~b}$ causes jaw malformation in zebrafish embryos. Mol Pharmacol. 2008;74(6):1544 53.

26. Planchart A, Mattingly CJ. 2,3,7,8-Tetrachlorodibenzo-p-dioxin upregulates FoxQ1b in zebrafish jaw primordium. Chem Res Toxicol. 2010;23(3):480-7.

27. Beis D, Stainier DY. In vivo cell biology: following the zebrafish trend. Trends Cell Biol. 2006;16(2):105-12.

28. Weber M, Huisken J. Light sheet microscopy for real-time developmental biology. Curr Opin Genet Dev. 2011;21(5):566-72.

29. Solnica-Krezel L, Schier AF, Driever W. Efficient recovery of ENU-induced mutations from the zebrafish germline. Genetics. 1994;136(4):1401-20.

30. Gaiano $\mathrm{N}$ et al. Insertional mutagenesis and rapid cloning of essential genes in zebrafish. Nature. 1996;383(6603):829-32.

31. Sander JD et al. Targeted gene disruption in somatic zebrafish cells using engineered TALENs. Nat Biotechnol. 2011;29(8):697-8.

32. Huang $P$ et al. Heritable gene targeting in zebrafish using customized TALENs. Nat Biotechnol. 2011;29(8):699-700.
33.• Hwang WY et al. Efficient genome editing in zebrafish using a CRISPR-Cas system. Nat Biotechnol. 2013;31(3):227-9. This study demonstrates the utility and application of the CRISPRCas system, which will greatly accelerate reverse genetic and mechanistic toxicological studies in the zebrafish.

34. Kettleborough RN et al. A systematic genome-wide analysis of zebrafish protein-coding gene function. Nature. 2013;496(7446): 494-7.

35. Fisher BE. 20 years of toxicology. Environ Health Perspect. 1998;106(10):A484-7.

36. Vogel SA, Roberts JA. Why the toxic substances control act needs an overhaul, and how to strengthen oversight of chemicals in the interim. Health Aff (Millwood). 2011;30(5):898-905.

37. Wilson MP, Schwarzman MR. Toward a new U.S. chemicals policy: rebuilding the foundation to advance new science, green chemistry, and environmental health. Environ Health Perspect. 2009;117(8):1202-9.

38. Van der Wielen A. REACH: next step to a sound chemicals management. J Expo Sci Environ Epidemiol. 2007;17 Suppl 1: S2-6.

39. Carpenter DO. Polychlorinated biphenyls (PCBs): routes of exposure and effects on human health. Rev Environ Health. 2006;21(1):1-23.

40. Lindemann K. On the prognosis and therapy of severe abnormalities of the extremities [in German]. Acta Orthop Scand. 1962;32: 298-306.

41. Lan SF, Starly B. Alginate based 3D hydrogels as an in vitro coculture model platform for the toxicity screening of new chemical entities. Toxicol Appl Pharmacol. 2011;256(1):62-72.

42. Godoy $\mathrm{P}$ et al. Recent advances in $2 \mathrm{D}$ and $3 \mathrm{D}$ in vitro systems using primary hepatocytes, alternative hepatocyte sources and non-parenchymal liver cells and their use in investigating mechanisms of hepatotoxicity, cell signaling and ADME. Arch Toxicol. 2013;87(8):1315-530.

43. DesRochers TM, Suter L, Roth A, Kaplan DL. Bioengineered 3D human kidney tissue, a platform for the determination of nephrotoxicity. PLoS One. 2013;8(3):e59219.

44. Padilla $\mathrm{S}$ et al. Zebrafish developmental screening of the ToxCast ${ }^{\mathrm{TM}}$ Phase I chemical library. Reprod Toxicol. 2012;33(2):174-87.

45. Truong $\mathrm{L}$ et al. Multidimensional in vivo hazard assessment using zebrafish. Toxicol Sci. 2014;137(1):212-33. This study is currently the most comprehensive evaluation of developmental toxicity for a large library of chemicals (1,060 compounds), and highlights a truly high-throughput application for zebrafish in vivo toxicity screening.

46. Bonventre JA, White LA, Cooper KR. Craniofacial abnormalities and altered wnt and mmp mRNA expression in zebrafish embryos exposed to gasoline oxygenates ETBE and TAME. Aquat Toxicol. 2012;120-121:45-53.

47. Soares AR et al. Ethanol exposure induces upregulation of specific microRNAs in zebrafish embryos. Toxicol Sci. 2012;127(1):1828.

48. Mattingly CJ, Hampton TH, Brothers KM, Griffin NE, Planchart A. Perturbation of defense pathways by low-dose arsenic exposure in zebrafish embryos. Environ Health Perspect. 2009;117(6):9817.

49. Praskova E et al. Acute toxicity of acetylsalicylic acid to juvenile and embryonic stages of Danio rerio. Neuroendocrinol Lett. 2012;33 Suppl 3:72-6.

50. Beker van Woudenberg A et al. A category approach to predicting the developmental (neuro) toxicity of organotin compounds: the value of the zebrafish (Danio rerio) embryotoxicity test (ZET). Reprod Toxicol. 2013;41:35-44.

51. Schock EN et al. The effects of carbaryl on the development of zebrafish (Danio rerio) embryos. Zebrafish. 2012;9(4):169-78. 
52. Hofsteen P, Plavicki J, Johnson SD, Peterson RE, Heideman W. Sox9b is required for epicardium formation and plays a role in TCDD-induced heart malformation in zebrafish. Mol Pharmacol. 2013;84(3):353-60.

53. Kim JY, Kim HH, Cho KH. Acute cardiovascular toxicity of sterilizers, PHMG, and PGH: severe inflammation in human cells and heart failure in zebrafish. Cardiovasc Toxicol. 2013;13(2): $148-60$.

54. Mathew LK, Simonich MT, Tanguay RL. AHR-dependent misregulation of Wnt signaling disrupts tissue regeneration. Biochem Pharmacol. 2009;77(4):498-507.

55. Hofsteen P, Mehta V, Kim MS, Peterson RE, Heideman W. TCDD inhibits heart regeneration in adult zebrafish. Toxicol Sci. 2013;132(1):211-21.

56. Coffin $\mathrm{AB}$ et al. Chemical screening for hair cell loss and protection in the zebrafish lateral line. Zebrafish. 2010;7(1):3-11.

57. Yang CT, Johnson SL. Small molecule-induced ablation and subsequent regeneration of larval zebrafish melanocytes. Development. 2006;133(18):3563-73.

58. Legler $\mathrm{J}$ et al. Effect-directed analysis of municipal landfill soil reveals novel developmental toxicants in the zebrafish Danio rerio. Environ Sci Technol. 2011;45(19):8552-8.

59. Fako VE, Furgeson DY. Zebrafish as a correlative and predictive model for assessing biomaterial nanotoxicity. Adv Drug Deliv Rev. 2009;61(6):478-86.

60. Chiu LL, Cunningham LL, Raible DW, Rubel EW, Ou HC. Using the zebrafish lateral line to screen for ototoxicity. J Assoc Res Otolaryngol. 2008;9(2):178-90.

61. Selderslaghs IW, Blust R, Witters HE. Feasibility study of the zebrafish assay as an alternative method to screen for developmental toxicity and embryotoxicity using a training set of 27 compounds. Reprod Toxicol. 2012;33(2):142-54.

62. Truong L, Mandrell D, Mandrell R, Simonich M, Tanguay RL. A rapid throughput approach identifies cognitive deficits in adult zebrafish from developmental exposure to polybrominated flame retardants. Neurotoxicology. 2014;43:134-42.

63. Antkiewicz DS, Peterson RE, Heideman W. Blocking expression of AHR2 and ARNT1 in zebrafish larvae protects against cardiac toxicity of 2,3,7,8-tetrachlorodibenzo-p-dioxin. Toxicol Sci. 2006;94(1):175-82.

64. Teraoka $\mathrm{H}$ et al. Induction of cytochrome $\mathrm{P} 4501 \mathrm{~A}$ is required for circulation failure and edema by 2,3,7,8-tetrachlorodibenzo-p-dioxin in zebrafish. Biochem Biophys Res Commun. 2003;304(2): 223-8.

65. Prasch AL et al. Aryl hydrocarbon receptor 2 mediates 2,3,7,8tetrachlorodibenzo-p-dioxin developmental toxicity in zebrafish. Toxicol Sci. 2003;76(1):138-50.

66. Goodale $\mathrm{BC}$ et al. AHR2 mutant reveals functional diversity of aryl hydrocarbon receptors in zebrafish. PLoS One. 2012;7(1):e29346.

67. Staudt D, Stainier D. Uncovering the molecular and cellular mechanisms of heart development using the zebrafish. Annu Rev Genet. 2012;46:397-418.

68. Hassel $\mathrm{D}$ et al. Nexilin mutations destabilize cardiac Z-disks and lead to dilated cardiomyopathy. Nat Med. 2009;15(11):1281-8.

69. Smith KA et al. Dominant-negative ALK2 allele associates with congenital heart defects. Circulation. 2009;119(24):3062-9.

70. Sedmera D et al. Functional and morphological evidence for a ventricular conduction system in zebrafish and Xenopus hearts. Am J Physiol Heart Circ Physiol. 2003;284(4):H1152-60.

71. Bakkers J. Zebrafish as a model to study cardiac development and human cardiac disease. Cardiovasc Res. 2011;91(2):279-88.

72. Incardona JP et al. Exxon Valdez to Deepwater Horizon: comparable toxicity of both crude oils to fish early life stages. Aquat Toxicol. 2013;142-143:303-16.

73. Incardona JP, Linbo TL, Scholz NL. Cardiac toxicity of 5-ring polycyclic aromatic hydrocarbons is differentially dependent on the aryl hydrocarbon receptor 2 isoform during zebrafish development. Toxicol Appl Pharmacol. 2011;257(2):242-9.

74. McGee SP, Konstantinov A, Stapleton HM, Volz DC. Aryl phosphate esters within a major PentaBDE replacement product induce cardiotoxicity in developing zebrafish embryos: potential role of the aryl hydrocarbon receptor. Toxicol Sci. 2013;133(1):144-56.

75. Plavicki J, Hofsteen P, Peterson RE, Heideman W. Dioxin inhibits zebrafish epicardium and proepicardium development. Toxicol Sci. 2013;131(2):558-67.

76. Waits ER, Nebert DW. Genetic architecture of susceptibility to PCB126-induced developmental cardiotoxicity in zebrafish. Toxicol Sci. 2011;122(2):466-75.

77. Chen J, Carney SA, Peterson RE, Heideman W. Comparative genomics identifies genes mediating cardiotoxicity in the embryonic zebrafish heart. Physiol Genomics. 2008;33(2):148-58.

78. Chen J. Impaired cardiovascular function caused by different stressors elicits a common pathological and transcriptional response in zebrafish embryos. Zebrafish. 2013;10(3):389-400.

79. Lee SH, Kim HR, Han RX, Oqani RK, Jin DI. Cardiovascular risk assessment of atypical antipsychotic drugs in a zebrafish model. J Appl Toxicol. 2013;33(6):466-70.

80. Cheng $\mathrm{H}$ et al. A novel preclinical strategy for identifying cardiotoxic kinase inhibitors and mechanisms of cardiotoxicity. Circ Res. 2011;109(12):1401-9.

81. Zhu JJ et al. Human cardiotoxic drugs delivered by soaking and microinjection induce cardiovascular toxicity in zebrafish. J Appl Toxicol. 2014;34(2):139-48.

82. Lal H, Kolaja KL, Force T. Cancer genetics and the cardiotoxicity of the therapeutics. J Am Coll Cardiol. 2013;61(3):267-74.

83. Choi WY et al. In vivo monitoring of cardiomyocyte proliferation to identify chemical modifiers of heart regeneration. Development. 2013;140(3):660-6.

84. Huang $\mathrm{CJ}, \mathrm{Tu} \mathrm{CT}$, Hsiao CD, Hsieh FJ, Tsai HJ. Germ-line transmission of a myocardium-specific GFP transgene reveals critical regulatory elements in the cardiac myosin light chain 2 promoter of zebrafish. Dev Dyn. 2003;228(1):30-40.

85. Kikuchi $\mathrm{K}$ et al. Primary contribution to zebrafish heart regeneration by gata4(+) cardiomyocytes. Nature. 2010;464(7288):601-5.

86. Poon KL, Liebling M, Kondrychyn I, Garcia-Lecea M, Korzh V. Zebrafish cardiac enhancer trap lines: new tools for in vivo studies of cardiovascular development and disease. Dev Dyn. 2010;239(3):914-26.

87. Kikuchi $\mathrm{K}$ et al. tcf21+ epicardial cells adopt non-myocardial fates during zebrafish heart development and regeneration. Development. 2011;138(14):2895-902.

88. McGrath P, Li CQ. Zebrafish: a predictive model for assessing drug-induced toxicity. Drug Discov Today. 2008;13(9-10):394 401.

89. Chu J, Sadler KC. New school in liver development: lessons from zebrafish. Hepatology. 2009;50(5):1656-63.

90. Goldstone JV et al. Identification and developmental expression of the full complement of cytochrome P450 genes in Zebrafish. BMC Genomics. 2010;11:643.

91. Kim KH et al. Cypla reporter zebrafish reveals target tissues for dioxin. Aquat Toxicol. 2013;134-135:57-65.

92. Mattingly CJ, McLachlan JA, Toscano Jr WA. Green fluorescent protein (GFP) as a marker of aryl hydrocarbon receptor (AhR) function in developing zebrafish (Danio rerio). Environ Health Perspect. 2001;109(8):845-9.

93. Noury P, Geffard O, Tutundjian R, Garric J. Non destructive in vivo measurement of ethoxyresorufin biotransformation by zebrafish prolarva: development and application. Environ Toxicol. 2006;21(4):324-31.

94. Power DM et al. Thyroid hormones in growth and development of fish. Comp Biochem Physiol C Toxicol Pharmacol. 2001;130(4): 447-59. 
95. Gorelick DA, Watson W, Halpern ME. Androgen receptor gene expression in the developing and adult zebrafish brain. Dev Dyn. 2008;237(10):2987-95.

96. Menuet A et al. Molecular characterization of three estrogen receptor forms in zebrafish: binding characteristics, transactivation properties, and tissue distributions. Biol Reprod. 2002;66(6):1881-92.

97. Levavi-Sivan B, Bogerd J, Mananos EL, Gomez A, Lareyre JJ. Perspectives on fish gonadotropins and their receptors. Gen Comp Endocrinol. 2010;165(3):412-37.

98. von Hofsten J, Olsson PE. Zebrafish sex determination and differentiation: involvement of FTZ-F1 genes. Reprod Biol Endocrinol. 2005;3:63.

99. Leal MC et al. Histological and stereological evaluation of zebrafish (Danio rerio) spermatogenesis with an emphasis on spermatogonial generations. Biol Reprod. 2009;81(1):177-87.

100. Clelland E, Peng C. Endocrine/paracrine control of zebrafish ovarian development. Mol Cell Endocrinol. 2009;312(1-2):42-52.

101. Griffin LB, January KE, Ho KW, Cotter KA, Callard GV. Morpholino-mediated knockdown of ERalpha, ERbetaa, and ERbetab mRNAs in zebrafish (Danio rerio) embryos reveals differential regulation of estrogen-inducible genes. Endocrinology. 2013;154(11):4158-69.

102. Bugel SM, White LA, Cooper KR. Inhibition of vitellogenin gene induction by 2,3,7,8-tetrachlorodibenzo-p-dioxin is mediated by aryl hydrocarbon receptor 2 (AHR2) in zebrafish (Danio rerio). Aquat Toxicol. 2013;126:1-8.

103. Petersen $\mathrm{K}$ et al. Transgenic (cyp19a1b-GFP) zebrafish embryos as a tool for assessing combined effects of oestrogenic chemicals. Aquat Toxicol. 2013;138-139:88-97.

104. Murk AJ, Legler J, Denison MS, et al. Chemical-activated luciferase gene expression (CALUX): a novel in vitro bioassay for Ah receptor active compounds in sediments and pore water. Fundam Appl Toxicol. 1996;33(1):149-60.

105. Heiden TK, Carvan 3rd MJ, Hutz RJ. Inhibition of follicular development, vitellogenesis, and serum 17beta-estradiol concentrations in zebrafish following chronic, sublethal dietary exposure to 2,3,7,8tetrachlorodibenzo-p-dioxin. Toxicol Sci. 2006;90(2):490-9.

106. King Heiden TC, Spitsbergen J, Heideman W, Peterson RE. Persistent adverse effects on health and reproduction caused by exposure of zebrafish to 2,3,7,8-tetrachlorodibenzo-p-dioxin during early development and gonad differentiation. Toxicol Sci. 2009;109(1):75-87.

107. Baker TR, Peterson RE, Heideman W. Using zebrafish as a model system for studying the transgenerational effects of dioxin. Toxicol Sci. 2014;138(2):403-11. This study demonstrates the utility of zebrafish to advance our understanding of transgenerational (epigenetic) inheritance, including parent-of-origin phenomenology.

108. Weger $\mathrm{M}$ et al. Real-time in vivo monitoring of circadian E-box enhancer activity: a robust and sensitive zebrafish reporter line for developmental, chemical and neural biology of the circadian clock. Dev Biol. 2013;380(2):259-73.

109. Suen MF et al. Assessments of the effects of nicotine and ketamine using tyrosine hydroxylase-green fluorescent protein transgenic zebrafish as biosensors. Biosens Bioelectron. 2013;42:177-85.

110. Sun Y, Dong Z, Jin T, et al. Imaging-based chemical screening reveals activity-dependent neural differentiation of pluripotent stem cells. Elife. 2013;2:e00508.

111. Raftery TD, Isales GM, Yozzo KL, Volz DC. High-content screening assay for identification of chemicals impacting spontaneous activity in zebrafish embryos. Environ Sci Technol. 2014;48(1):804-10.

112. Zhang X, Gong Z. Fluorescent transgenic zebrafish $\operatorname{Tg}(n k x 2.2 a$ : mEGFP) provides a highly sensitive monitoring tool for neurotoxins. PLoS One. 2013;8(2):e55474.

113. Kanungo J, Lantz S, Paule MG. In vivo imaging and quantitative analysis of changes in axon length using transgenic zebrafish embryos. Neurotoxicol Teratol. 2011;33(6):618-23.
114. Makhija DT, Jagtap AG. Studies on sensitivity of zebrafish as a model organism for Parkinson's disease: comparison with rat model. J Pharmacol Pharmacother. 2014;5(1):39-46.

115. Da Costa MM, Allen CE, Higginbottom A, et al. A new zebrafish model produced by TILLING of SOD1-related amyotrophic lateral sclerosis replicates key features of the disease and represents a tool for in vivo therapeutic screening. Dis Model Mech. 2014;7(1): $73-81$.

116. Armstrong GA, Drapeau P. Loss and gain of FUS function impair neuromuscular synaptic transmission in a genetic model of ALS. Hum Mol Genet. 2013;22(21):4282-92.

117. Ciura S, Lattante S, Le Ber I, et al. Loss of function of C9orf72 causes motor deficits in a zebrafish model of amyotrophic lateral sclerosis. Ann Neurol. 2013;74(2):180-7.

118. Schmid B et al. Loss of ALS-associated TDP-43 in zebrafish causes muscle degeneration, vascular dysfunction, and reduced motor neuron axon outgrowth. Proc Natl Acad Sci U S A. 2013;110(13):4986-91.

119. McGown A et al. Early interneuron dysfunction in ALS: insights from a mutant sod1 zebrafish model. Ann Neurol. 2013;73(2): 246-58.

120. Wager K, Russell C. Mitophagy and neurodegeneration: the zebrafish model system. Autophagy. 2013;9(11):1693-709.

121. Irons TD, MacPhail RC, Hunter DL, Padilla S. Acute neuroactive drug exposures alter locomotor activity in larval zebrafish. Neurotoxicol Teratol. 2010;32(1):84-90.

122. Sun EY, Josephson L, Kelly KA, Weissleder R. Development of nanoparticle libraries for biosensing. Bioconjug Chem. 2006;17(1):109-13.

123. Harper SL, Carriere JL, Miller JM, et al. Systematic evaluation of nanomaterial toxicity: utility of standardized materials and rapid assays. ACS Nano. 2011;5(6):4688-97.

124. Tang $\mathrm{S}$ et al. Cadmium sulfate and CdTe-quantum dots alter DNA repair in zebrafish (Danio rerio) liver cells. Toxicol Appl Pharmacol. 2013;272(2):443-52.

125. Zhang $\mathrm{W}$ et al. Toxicity assessment of zebrafish following exposure to CdTe QDs. J Hazard Mater. 2012;213-214:413-20.

126. Kim KT, Zaikova T, Hutchison JE, Tanguay RL. Gold nanoparticles disrupt zebrafish eye development and pigmentation. Toxicol Sci. 2013;133(2):275-88.

127. Bar-Ilan O, Albrecht RM, Fako VE, Furgeson DY. Toxicity assessments of multisized gold and silver nanoparticles in zebrafish embryos. Small. 2009;5(16):1897-910.

128. Christen V, Capelle M, Fent K. Silver nanoparticles induce endoplasmatic reticulum stress response in zebrafish. Toxicol Appl Pharmacol. 2013;272(2):519-28.

129. Griffitt RJ, Lavelle CM, Kane AS, Denslow ND, Barber DS Chronic nanoparticulate silver exposure results in tissue accumulation and transcriptomic changes in zebrafish. Aquat Toxicol. 2013;130-131:192-200.

130. Lee KJ et al. In vivo quantitative study of sized-dependent transport and toxicity of single silver nanoparticles using zebrafish embryos. Chem Res Toxicol. 2012;25(5):1029-46.

131. Powers CM, Slotkin TA, Seidler FJ, Badireddy AR, Padilla S. Silver nanoparticles alter zebrafish development and larval behavior: distinct roles for particle size, coating and composition. Neurotoxicol Teratol. 2011;33(6):708-14.

132. Griffitt RJ, Hyndman K, Denslow ND, Barber DS. Comparison of molecular and histological changes in zebrafish gills exposed to metallic nanoparticles. Toxicol Sci. 2009;107(2):404-15.

133. Duan J, Yu Y, Li Y, Yu Y, Sun Z. Cardiovascular toxicity evaluation of silica nanoparticles in endothelial cells and zebrafish model. Biomaterials. 2013;34(23):5853-62.

134. Xiong D, Fang T, Yu L, Sima X, Zhu W. Effects of nano-scale $\mathrm{TiO} 2, \mathrm{ZnO}$ and their bulk counterparts on zebrafish: acute toxicity, 
oxidative stress and oxidative damage. Sci Total Environ. 2011;409(8):1444-52.

135. Clemente Z, Castro VL, Moura MA, Jonsson CM, Fraceto LF. Toxicity assessment of $\mathrm{TiO}(2)$ nanoparticles in zebrafish embryos under different exposure conditions. Aquat Toxicol. 2014;147: 129-39.

136. Mandrell $\mathrm{D}$ et al. Automated zebrafish chorion removal and single embryo placement: optimizing throughput of zebrafish developmental toxicity screens. J Lab Autom. 2012;17(1):66-74.

137. Peravali $\mathrm{R}$ et al. Automated feature detection and imaging for high-resolution screening of zebrafish embryos. Biotechniques. 2011;50(5):319-24.

138. Saydmohammed M, Vollmer LL, Onuoha EO, Vogt A, Tsang M. A high-content screening assay in transgenic zebrafish identifies two novel activators of fgf signaling. Birth Defects Res C Embryo Today. 2011;93(3):281-7.
139. Yozzo KL, Isales GM, Raftery TD, Volz DC. High-content screening assay for identification of chemicals impacting cardiovascular function in zebrafish embryos. Environ Sci Technol. 2013;47(19): 11302-10. This manuscript highlights recent advancements in high-content imaging and automated detection of cardiotoxicants in the zebrafish embryo model.

140. Paul SM et al. How to improve R\&D productivity: the pharmaceutical industry's grand challenge. Nat Rev Drug Discov. 2010;9(3):203-14.

141. Zon LI, Peterson RT. In vivo drug discovery in the zebrafish. Nat Rev Drug Discov. 2005;4(1):35-44.

142. North TE et al. Prostaglandin E2 regulates vertebrate haematopoietic stem cell homeostasis. Nature. 2007;447(7147):1007-11.

143. Ball $\mathrm{J}$ et al. Fishing for teratogens: a consortium effort for a harmonized zebrafish developmental toxicology assay. Toxicol Sci. 2014;139(1):210-9. 